\title{
C-class Functions and Remarks on Fixed Points of Weakly Compatible Mappings in G-Metric Spaces Satisfying Common Limit Range Property
}

\author{
A. H. Ansari, D. Dolićanin-Đekić, F. Gu, B. Z. Popović,
}

\begin{abstract}
In this paper, using the contexts of C-class functions and common limit range property, common fixed point result for some operators are obtained.
\end{abstract}

Keywords: Generalized metric space; Common fixed point; Generalized weakly G-contraction; Weakly compatible mappings.

\section{Introduction and preliminaries}

The study of common fixed point theorems satisfying contractive conditions has a wide range of applications in different areas such as, variational and linear inequality problems, optimization and parameterize estimation problems and many others. One of the simplest and most useful results in the fixed point theory is the Banach-Caccioppoli contraction principle. This theorem provides a technique for solving a variety of applied problems in mathematical sciences and engineering.

Banach contraction principle has been generalized in different spaces by mathematicians over the years. Mustafa and Sims [18] proposed a new class of generalized metric spaces, which are called as G-metric spaces. In this type of spaces a non-negative real number is assigned to every triplet of elements. Many mathematicians studied extensively various results on G-metric spaces by using the concept of weak commutativity, compatibility, non-compatibility and weak compatibility for single valued mappings satisfying different contractive conditions (cf. [2]-[8], [10]-[12], [15], [17]-[29]).

Manuscript received April 3, 2016; accepted August 15, 2016.

A. H. Ansari is with the Department of Mathematics, Islamic Azad University, Karaj, Iran; D. DolićaninĐekić is with the Faculty of Technical Science, Kosovska Mitrovica, Serbia; F. Gu is with the Institute of Applied Mathematics and Department of Mathematics, Hangzhou Normal University, Hangzhou, China; B. Z. Popović is with the Faculty of Science, University of Kragujevac, Serbia. 
Branciari [9] obtained a fixed point result for a single mapping satisfying an analogue of Banach's contraction principle for an integral type inequality. This influenced many authors, and consequently, a number of new results in this line followed (see, for example [7]). Later on, Aydi [7] proved an integral type fixed point theorem for two self mappings and extended the results of Brianciari [9] to the class of G-metric spaces. The first fixed point theorem without any continuity requirement was proved by Abbas and Rhoades [4] in which they utilized the notion of non-commuting mappings for the existence of fixed points. Shatanawi et al. [29] proved some interesting fixed point results by using $\varphi$-contractive condition and generalized the results of Abbas and Rhoades [4]. Most recently, Mustafa et al. [25] defined the notion of the property $(E . A)$ in G-metric space and proved some fixed point results.

In this paper, firstly we prove an integral type fixed point theorem for a pair of weakly compatible mappings in G-metric space satisfying the common limit range property which is initiated by Sintunavarat and Kumam [30]. We extend our main result to two finite families of self mappings by using the notion of pairwise commuting. We also present some fixed point results in $G$-metric spaces satisfying $\phi$-contractions. Some related examples are furnished to support our results.

Now we give preliminaries and basic definitions which are used throughout the paper.

Definition 1 [18] Let $X$ be a nonempty set, and let $G: X \times X \times X \longrightarrow[0, \infty)$ be a function satisfying the following axioms:

(G1) $G(x, y, z)=0$ if $x=y=z$;

(G2) $0<G(x, x, y)$, for all $x, y \in X$ with $x \neq y$;

(G3) $G(x, x, y) \leq G(x, y, z)$,for all $x, y, z \in X$ with $z \neq y$;

(G4) $G(x, y, z)=G(x, z, y)=G(y, z, x)=\ldots$ (symmetry in all three variables);

(G5) $G(x, y, z) \leq G(x, a, a)+G(a, y, z)$ for all $x, y, z, a \in X$, (rectangle inequality)

then the function $G$ is called a generalized metric, or, more specifically a $G$-metric on $X$ and the pair $(X, G)$ is called a $G$-metric space.

It is known that the function $G(x, y, z)$ on a $G$-metric space $X$ is jointly continuous in all three of its variables, and $G(x, y, z)=0$ if and only if $x=y=z$; see [18] for more details and the reference therein.

Definition 2 [18] Let $(X, G)$ be a G-metric space, and let $\left\{x_{n}\right\}$ be a sequence of points in $X$, a point $x$ in $X$ is said to be the limit of the sequence $\left\{x_{n}\right\}$ if $\lim _{m, n \rightarrow \infty} G\left(x, x_{n}, x_{m}\right)=0$, and one says that sequence $\left\{x_{n}\right\}$ is $G$-convergent to $x$.

Thus, if $x_{n} \rightarrow x$ in a $G$-metric space $(X, G)$, then for any $\varepsilon>0$, there exists $N \in \mathbb{N}$ (throughout this paper we mean by $\mathbb{N}$ the set of all natural numbers) such that $G\left(x, x_{n}, x_{m}\right)<$ $\varepsilon$, for all $n, m \geq N$ 
Proposition 1 [18] Let $(X, G)$ be a G-metric space, then the following are equivalent:

(1) $\left\{x_{n}\right\}$ is G-convergent to $x$.

(2) $G\left(x_{n}, x_{n}, x\right) \rightarrow 0$ as $n \rightarrow \infty$.

(3) $G\left(x_{n}, x, x\right) \rightarrow 0$ as $n \rightarrow \infty$.

(4) $G\left(x_{n}, x_{m}, x\right) \rightarrow 0$ as $n, m \rightarrow \infty$.

Definition 3 [18] Let $(X, G)$ be a G-metric space. A sequence $\left\{x_{n}\right\}$ is called G-Cauchy sequence if, for each $\varepsilon>0$, there exists $N \in \mathbb{N}$ such that $G\left(x_{n}, x_{m}, x_{l}\right)<\varepsilon$ for all $n, m, l \geq N$; i.e., if $G\left(x_{n}, x_{m}, x_{l}\right) \rightarrow 0$ as $n, m, l \rightarrow \infty$.

Definition 4 [18] A G-metric space $(X, G)$ is said to be G-complete (or a complete Gmetric space) if every $G$-Cauchy sequence in $(X, G)$ is $G$-convergent in $X$.

Proposition 2 [18] Let $(X, G)$ be a G-metric space. Then the following are equivalent.

(1) The sequence $\left\{x_{n}\right\}$ is $G$-Cauchy.

(2) For every $\varepsilon>0$, there exists $k \in \mathbb{N}$ such that $G\left(x_{n}, x_{m}, x_{m}\right)<\varepsilon$, for all $n, m \geq k$.

Proposition 3 [18] Let $(X, G)$ be a G-metric space. Then the function $G(x, y, z)$ is jointly continuous in all three of its variables.

Proposition 4 [18] Let $(X, G)$ be a G-metric space. Then, for all $x, y$ in $X$ it follows that $G(x, y, y) \leq 2 G(y, x, x)$.

Definition 5 [13] Let $f$ and $g$ be self maps of a set $X$. If $w=f x=g x$ for some $x$ in $X$, then $x$ is called a coincidence point of $f$ and $g$, and $w$ is called point of coincidence of $f$ and $g$.

Definition 6 [13] Two self mappings $f$ and $g$ on $X$ are said to be weakly compatible if they commute at coincidence points.

Definition 7 [8] Let $X$ be a G-metric space. Self mappings $f$ and $g$ on $X$ are said to satisfy the $G-(E . A)$ property if there exists a sequence $\left\{x_{n}\right\}$ in $X$ such that $\left\{f x_{n}\right\}$ and $\left\{g x_{n}\right\}$ are $G$-convergent to some $t \in X$.

Definition 8 ([8], [30]) A pair $(f, g)$ of self mappings of a G-metric space $(X, G)$ is said to satisfy the (CLRg) property if there exists a sequence $\left\{x_{n}\right\}$ such that $\left\{f x_{n}\right\}$ and $\left\{g x_{n}\right\}$ $G$-converge to gt for some $t \in X$, that is,

$$
\lim _{n \rightarrow \infty} G\left(f x_{n}, f x_{n}, g t\right)=\lim _{n \rightarrow \infty} G\left(g x_{n}, g x_{n}, g t\right)=0 .
$$

Definition 9 A pair $(f, g)$ of self mappings of a $G$-metric space $(X, G)$ is said to satisfy the $(L R g)$ property if there exists a sequence $\left\{x_{n}\right\}$ such that $\left\{f x_{n}\right\}$ and $\left\{g x_{n}\right\} G$-converge to gt for some $t \in f(X) \cap g(X)$, that is,

$$
\lim _{n \rightarrow \infty} G\left(f x_{n}, f x_{n}, g t\right)=\lim _{n \rightarrow \infty} G\left(g x_{n}, g x_{n}, g t\right)=0 .
$$


Definition 10 Self mappings $f$ and $g$ of a G-metric space $(X, G)$ are said to be compatible if $\lim _{n \rightarrow \infty} G\left(f g x_{n}, g f x_{n}, g f x_{n}\right)=0$ and $\lim _{n \rightarrow \infty} G\left(g f x_{n}, f g x_{n}, f g x_{n}\right)=0$, whenever $\left\{x_{n}\right\}$ is a sequence in $X$ such that $\lim _{n \rightarrow} f x_{n}=\lim _{n \rightarrow \infty} g x_{n}=t$, for some $t \in X$.

Khan et al. [16] introduced the concept of altering distance function that is a control function employed to alter the metric distance between two points enabling one to deal with relatively new classes of fixed point problems. Here, we consider the following notion.

Definition 11 [16] The function $\psi:[0,+\infty) \rightarrow[0,+\infty)$ is called an altering distance function if the following properties are satisfied:

(1) $\psi$ is continuous and increasing;

(2) $\psi(t)=0$ if and only if $t=0$.

We denote $\Psi$ set all of altering distance function.

In 2014 the concept of $C$-class functions (see Definition 12) was introduced by A. H. Ansari in [1] that is able to notice that can see in numbers (1), (2), (9) and (15) from Example 1.

Definition 12 A mapping $F:[0, \infty)^{2} \rightarrow \mathbb{R}$ is called $C$-class function if it is continuous and satisfies following axioms:

(1) $F(s, t) \leq s$;

(2) $F(s, t)=s$ implies that either $s=0$ or $t=0$; for all $s, t \in[0, \infty)$.

Note for some $F$ we have that $F(0,0)=0$.

We denote $C$-class functions as $\mathscr{C}$.

Example 1 The following functions $F:[0, \infty)^{2} \rightarrow \mathbb{R}$ are elements of $\mathscr{C}$, for all $s, t \in[0, \infty)$ :

(1) $F(s, t)=s-t, F(s, t)=s \Rightarrow t=0$;

(2) $F(s, t)=m s, 0<m<1, F(s, t)=s \Rightarrow s=0$;

(3) $F(s, t)=\frac{s}{(1+t)^{r}} ; r \in(0, \infty), F(s, t)=s \Rightarrow s=0$ or $t=0$;

(4) $F(s, t)=\log \left(t+a^{s}\right) /(1+t), a>1, F(s, t)=s \Rightarrow s=0$ or $t=0$;

(5) $F(s, t)=\ln \left(1+a^{s}\right) / 2, a>e, F(s, 1)=s \Rightarrow s=0$;

(6) $F(s, t)=(s+l)^{\left(1 /(1+t)^{r}\right)}-l, l>1, r \in(0, \infty), F(s, t)=s \Rightarrow t=0$;

(7) $F(s, t)=s \log _{t+a} a, a>1, F(s, t)=s \Rightarrow s=0$ or $t=0$;

(8) $F(s, t)=s-\left(\frac{1+s}{2+s}\right)\left(\frac{t}{1+t}\right), F(s, t)=s \Rightarrow t=0$;

(9) $F(s, t)=s \beta(s), \beta:[0, \infty) \rightarrow[0,1), F(s, t)=s \Rightarrow s=0$;

(10) $F(s, t)=s-\frac{t}{k+t}, F(s, t)=s \Rightarrow t=0$;

(11) $F(s, t)=s-\varphi(s), F(s, t)=s \Rightarrow s=0$, here $\varphi:[0, \infty) \rightarrow[0, \infty)$ is a continuous function such that $\varphi(t)=0 \Leftrightarrow t=0$;

(12) $F(s, t)=\operatorname{sh}(s, t), F(s, t)=s \Rightarrow s=0$, here $h:[0, \infty) \times[0, \infty) \rightarrow[0, \infty)$ is a continuous function such that $h(t, s)<1$ for all $t, s>0$; 
(13) $F(s, t)=s-\left(\frac{2+t}{1+t}\right) t, F(s, t)=s \Rightarrow t=0$.

(14) $F(s, t)=\sqrt[n]{\ln \left(1+s^{n}\right)}, F(s, t)=s \Rightarrow s=0$.

(15) $F(s, t)=\phi(s), F(s, t)=s \Rightarrow s=0$, here $\phi:[0, \infty) \rightarrow[0, \infty)$ is a upper semicontinuous function such that $\phi(0)=0$, and $\phi(t)<t$ for $t>0$,

(16) $F(s, t)=\frac{s}{(1+s)^{r}} ; r \in(0, \infty), F(s, t)=s \Rightarrow s=0$.

Problem: Whether can say that for all $F$ we have $F(0,0)=0$ ?

Definition 13 An ultra altering distance function is a continuous, nondecreasing mapping $\varphi:[0, \infty) \rightarrow[0, \infty)$ such that $\varphi(t)>0$ if $t>0$ and $\varphi(0) \geq 0$.

Remark 1 We denote $\Phi_{u}$ set all of ultra altering distance functions.

In the sequel let $\Phi$ be the set of all functions $\omega$ such that $\omega:[0,+\infty) \rightarrow[0,+\infty)$ is a nondecreasing function with $\lim _{n \rightarrow+\infty} \omega^{n}(t)=0$ for all $t \in(0,+\infty)$. If $\omega \in \Phi$, then $\omega$ is called a $\Phi$-mapping. If $\omega$ is a $\Phi$-mapping, then it is easy matter to show that:

1. $\omega(t)<t$ for all $t \in(0,+\infty)$,

2. $\omega(0)=0$.

\section{Main results}

We start with the following theorem.

Theorem 1 Let $(X, G)$ be a G-metric space and the pair $(f, g)$ of self mappings is weakly compatible such that

$$
\int_{0}^{\psi(G(f x, f y, f z))} \varphi(t) d t \leq F\left(\int_{0}^{\psi(L(x, y, z))} \varphi(t) d t, \int_{0}^{\phi(L(x, y, z))} \varphi(t) d t\right),
$$

for all $x, y, z \in X, F:[0, \infty)^{2} \rightarrow \mathbb{R}$ is a C-class, $\psi \in \Psi, \phi \in \Phi_{u}$ and $\varphi:[0,+\infty) \rightarrow[0,+\infty)$ is a Lebesgue integrable mapping which is summable, non-negative and such that for each $\varepsilon>0, \int_{0}^{\varepsilon} \varphi(t) d t>0$ where

$$
L(x, y, z)=\max \{G(g x, g y, g z), G(g x, f x, f x), G(g y, f y, f y), G(g z, f z, f z)\},
$$

or

$$
L(x, y, z)=\max \{G(g x, g y, g z), G(g x, g x, f x), G(g y, g y, f y), G(g z, g z, f z)\} .
$$

If the pair $(f, g)$ satisfies the (CLRg) property then $f$ and $g$ have a unique common fixed point in $X$. 
Proof Since the pair $(f, g)$ satisfies the $\left(C L R_{g}\right)$ property, then there exists a sequence $\left\{x_{n}\right\}$ in $X$ such that $\lim _{n \rightarrow \infty} f x_{n}=\lim _{n \rightarrow \infty} g x_{n}=g u$ for some $u \in X$. We show that $f u=g u$. On using inequality (1), we get

$$
\int_{0}^{\psi\left(G\left(f x_{n}, f x_{n}, f u\right)\right)} \varphi(t) d t \leq F\left(\int_{0}^{\psi\left(L\left(x_{n}, x_{n}, u\right)\right)} \varphi(t) d t, \int_{0}^{\phi\left(L\left(x_{n}, x_{n}, u\right)\right)} \varphi(t) d t\right),
$$

where

$$
\begin{gathered}
L\left(x_{n}, x_{n}, u\right)=\max \left\{G\left(g x_{n}, g x_{n}, g u\right), G\left(g x_{n}, f x_{n}, f x_{n}\right),\right. \\
\left.G\left(g x_{n}, f x_{n}, f x_{n}\right), G(g u, f u, f u)\right\} .
\end{gathered}
$$

Taking limit as $n \rightarrow+\infty$ in (4), we have

$$
\int_{0}^{\psi(G(g u, g u, f u))} \varphi(t) d t \leq F\left(\int_{0}^{\psi(G(g u, f u, f u))} \varphi(t) d t, \int_{0}^{\phi(G(g u, f u, f u))} \varphi(t) d t\right) .
$$

Similarly, one can obtain

$$
\int_{0}^{\psi(G(g u, f u, f u))} \varphi(t) d t \leq F\left(\int_{0}^{\psi(G(g u, g u, f u))} \varphi(t) d t, \int_{0}^{\phi(G(g u, g u, f u))} \varphi(t) d t\right) .
$$

From (5) and (6), we have

$$
\begin{aligned}
\int_{0}^{\psi(G(g u, g u, f u))} \varphi(t) d t & \leq \int_{0}^{\psi(G(g u, f u, f u))} \varphi(t) d t \\
& \leq F\left(\int_{0}^{\psi G(g u, g u, f u))} \varphi(t) d t, \int_{0}^{\phi(G(g u, g u, f u))} \varphi(t) d t\right) .
\end{aligned}
$$

So,

$$
\int_{0}^{\psi(G(g u, g u, f u))} \varphi(t) d t=0 \quad \text { or } \quad \int_{0}^{\phi(G(g u, g u, f u))} \varphi(t) d t=0,
$$

therefore $\psi(G(g u, g u, f u))=0$ or $\phi(G(g u, g u, f u))=0$. Thus $G(g u, g u, f u)=0$, that is, $f u=g u$. Suppose that $w=f u=g u$. Since the pair $(f, g)$ is weakly compatible and $w=$ $f u=g u$, therefore $f w=f g u=g f u=g w$. Finally, we prove that $w=f w$. Inequality (1) implies

$$
\int_{0}^{\psi(G(f w, f w, f u))} \varphi(t) d t \leq F\left(\int_{0}^{\psi(L(w, w, u))} \varphi(t) d t, \int_{0}^{\phi(L(w, w, u))} \varphi(t) d t\right),
$$

where

$$
\begin{aligned}
L(w, w, u) & =\max \{G(g w, g w, g u), G(g w, f w, f w), G(g w, f w, f w), G(g u, f u, f u)\} \\
& =\max \{G(f w, f w, w), G(f w, f w, f w), G(f w, f w, f w), G(w, w, w)\} \\
& =G(f w, f w, w) .
\end{aligned}
$$


Therefore (7) implies

$$
\int_{0}^{\psi(G(f w, f w, w))} \varphi(t) d t \leq F\left(\int_{0}^{\psi(G(f w, f w, w))} \varphi(t) d t, \int_{0}^{\phi(G(f w, f w, w))} \varphi(t) d t\right)
$$

so,

$$
\int_{0}^{\psi(G(f w, f w, w))} \varphi(t) d t=0 \quad \text { or } \quad \int_{0}^{\phi(G(f w, f w, w))} \varphi(t) d t=0 .
$$

Therefore $\psi(G(f w, f w, w))=0$ or $\phi(G(f w, f w, w))=0$, thus $G(f w, f w, w)=0$, that is, $w=f w$. Therefore, $w$ is a common fixed point of the mappings $f$ and $g$. The proof is similar for condition (3), hence the details are omitted. Uniqueness of the common fixed point is easy consequences of inequalities (1)-(7).

With choice $F(s, t)=s-t$ in Theorem 1 we have the following corollary.

Corollary 1 Let $(X, G)$ be a G-metric space and the pair $(f, g)$ of self mappings is weakly compatible such that

$$
\int_{0}^{\psi(G(f x, f y, f z)} \varphi(t) d t \leq \int_{0}^{\psi(L(x, y, z))} \varphi(t) d t-\int_{0}^{\phi(L(x, y, z))} \varphi(t) d t
$$

for all $x, y, z \in X, \psi \in \Psi, \phi \in \Phi_{u}$ and $\varphi:[0,+\infty) \rightarrow[0,+\infty)$ is a Lebesgue integrable mapping which is summable, non-negative and such that for each $\varepsilon>0, \int_{0}^{\varepsilon} \varphi(t) d t>0$, where

$$
L(x, y, z)=\max \{G(g x, g y, g z), G(g x, f x, f x), G(g y, f y, f y), G(g z, f z, f z)\},
$$

or

$$
L(x, y, z)=\max \{G(g x, g y, g z), G(g x, g x, f x), G(g y, g y, f y), G(g z, g z, f z)\} .
$$

If the pair $(f, g)$ satisfies the (CLRg) property, then $f$ and $g$ have a unique common fixed point in $X$.

With choice $F(s, t)=k s, 0 \leq k<1$ in Theorem 1 we have the following corollary.

Corollary 2 Let $(X, G)$ be a G-metric space and the pair $(f, g)$ of self mappings is weakly compatible such that

$$
\int_{0}^{\psi(G(f x, f y, f z))} \varphi(t) d t \leq k \int_{0}^{\psi(L(x, y, z))} \varphi(t) d t,
$$


for all $x, y, z \in X, 0 \leq k<1, \psi \in \Psi$ and $\varphi:[0,+\infty) \rightarrow[0,+\infty)$ is a Lebesgue integrable mapping which is summable, non-negative and such that for each $\varepsilon>0, \int_{0}^{\varepsilon} \varphi(t) d t>0$, where

$$
L(x, y, z)=\max \{G(g x, g y, g z), G(g x, f x, f x), G(g y, f y, f y), G(g z, f z, f z)\}
$$

or

$$
L(x, y, z)=\max \{G(g x, g y, g z), G(g x, g x, f x), G(g y, g y, f y), G(g z, g z, f z)\} .
$$

If the pair $(f, g)$ satisfies the (CLRg) property then $f$ and $g$ have a unique common fixed point in $X$.

With choice $F(s, t)=s \beta(s), \beta:[0, \infty) \rightarrow[0,1)$, in Theorem 1 we have the following corollary.

Corollary 3 Let $(X, G)$ be a G-metric space and the pair $(f, g)$ of self mappings is weakly compatible such that

$$
\int_{0}^{G(f x, f y, f z)} \varphi(t) d t \leq k \int_{0}^{G(g x, g y, g z)} \varphi(t) d t
$$

for all $x, y, z \in X, 0 \leq k<1$, and $\varphi:[0,+\infty) \rightarrow[0,+\infty)$ is a Lebesgue integrable mapping which is summable, non-negative and such that for each $\varepsilon>0, \int_{0}^{\varepsilon} \varphi(t) d t>0$. If the pair $(f, g)$ satisfies the $(C L R g)$ property then $f$ and $g$ have a unique common fixed point in $X$.

With choice $F(s, t)=\omega(s)$, here $\omega:[0, \infty) \rightarrow[0, \infty)$ is a continuous function such that $\omega(0)=0$, and $\omega(t)<t$ for $t>0$, in Theorem 1 we have the following corollary.

Corollary 4 Let $(X, G)$ be a G-metric space and the pair $(f, g)$ of self mappings is weakly compatible such that

$$
\int_{0}^{\psi(G(f x, f y, f z))} \varphi(t) d t \leq \omega\left(\int_{0}^{\psi(L(x, y, z))} \varphi(t) d t\right)
$$

for all $x, y, z \in X, \omega \in \Phi, \psi \in \Psi$, and $\varphi:[0,+\infty) \rightarrow[0,+\infty)$ is a Lebesgue integrable mapping which is summable, non-negative and such that for each $\varepsilon>0, \int_{0}^{\varepsilon} \varphi(t) d t>0$, where

$$
L(x, y, z)=\max \{G(g x, g y, g z), G(g x, f x, f x), G(g y, f y, f y), G(g z, f z, f z)\}
$$

or

$$
L(x, y, z)=\max \{G(g x, g y, g z), G(g x, g x, f x), G(g y, g y, f y), G(g z, g z, f z)\} .
$$

If the pair $(f, g)$ satisfies the (CLRg) property then $f$ and $g$ have a unique common fixed point in $X$. 
With choice $\psi(t)=t$, in Corollary 4 we have the following corollary.

Corollary 5 [8] Let $(X, G)$ be a G-metric space and the pair $(f, g)$ of self mappings is weakly compatible such that

$$
\int_{0}^{G(f x, f y, f z)} \varphi(t) d t \leq \omega\left(\int_{0}^{L(x, y, z)} \varphi(t) d t\right)
$$

for all $x, y, z \in X, \omega \in \Phi$, and $\varphi:[0,+\infty) \rightarrow[0,+\infty)$ is a Lebesgue integrable mapping which is summable, non-negative and such that for each $\varepsilon>0, \int_{0}^{\varepsilon} \varphi(t) d t>0$, where

$$
L(x, y, z)=\max \{G(g x, g y, g z), G(g x, f x, f x), G(g y, f y, f y), G(g z, f z, f z)\},
$$

or

$$
L(x, y, z)=\max \{G(g x, g y, g z), G(g x, g x, f x), G(g y, g y, f y), G(g z, g z, f z)\} .
$$

If the pair $(f, g)$ satisfies the (CLRg) property, then $f$ and $g$ have a unique common fixed point in $X$.

\section{References}

[1] A. H. ANSARI, Note on " $\varphi-\psi$-contractive type mappings and related fixed point", The 2 nd Regional Conference on Mathematics and Applications, PNU, September 2014, 77-380

[2] M. ABBAS, S. H. KHAN AND T. NAZIR, Common fixed points of $R$-weakly commuting maps in generalized metric spaces, Fixed Point Theory Appl. 2011, 2011:41

[3] M. ABbas, T. NAZIR AND S. RADENović, Common fixed point of generalized weakly contractive maps in partially ordered G-metric spaces, Appl. Math. Comput. 218 (18) (2012), 9383-9395

[4] M. ABBAS AND B. E. RHOADES, Common fixed point results for noncommuting mappings without continuity in generalized metric spaces, Appl. Math. Comput. 215 (1) (2009) 262-269

[5] M. ABbas, S. H. Khan, T. NAZIR, Common fixed points of R-weakly commuting maps in generalized metric spaces, Fixed Point Theory Appl.. 2011, Article ID 784595, 11 pages

[6] M. ABBAS, T NAZIR, D. DJORIĆ, Common fixed point of mappings satisfying (E.A) property in generalized metric spaces, Appl. Math. Comput. 218 (14), 7665-7670 (2012)

[7] H. AYDI, A common fixed point of integral type contraction in generalized metric spaces, J. Adv. Math. Stud. 5 (1) (2012), 111-117.

[8] H. Aydi, S. ChaUhan AND S. RADENOVIĆ, Fixed point od weakly compatible mappings in G-metric spaces satisfying common limit range property, Facta Univerzitatis (Niš) Ser. Math. Inform. Vol. 28, No 2 (2013), 197-210

[9] A. BRANCIARI, A fixed point theorem for mappings satisfying a general contractive condition of integral type, Int. J. Math. Math. Sci. 29 (9) (2002), 531-536 
[10] F. GU, Y. YIN, Common fixed point for three pairs of self-maps satisfying common (E.A) property in generalized metric spaces, Abstr. Appl. Anal.. 2013, Article ID 808092, 13 pages

[11] F. Gu, Common fixed point theorems for six mappings in generalized metric spaces, Abstr. Appl. Anal.. 2012, Article ID 379212, 21 pages

[12] F. Gu AND W. Shatanawi, Common fixed point for generalized weakly G-contraction mappings satisfying common (E.A) property in G-metric spaces, Fixed Point Theory Appl. 2013, 2013:309

[13] M. ABBAS, G. JUNGCK, Common fixed point results for non-commuting mappings without continuity in cone metric spaces, J. Math. Anal. Appl., 341 (2008) 416-420.

[14] Z. Kadelburg, H. K. NaŠINE, AND S. Radenović, Common coupled fixed point results in partially ordered $G$-metric spaces, Bulletinof Mathematical Analysis and Applications, Volume 4, Issue 2 (2012), Pages 51-63

[15] A. Kaewcharoen, Common fixed points for four mappings in G-metric spaces, Int. J. Math. Anal. 6 (47), 2345-2356 (2012)

[16] M. S. Khan, M. Swaleh, S. SesSa, Fixed point theorems by altering distances between the points, Bull. Aust. Math. Soc. 30, 1-9 (1984)

[17] W. Long, M. Abbas, T. Nazir, S. Radenović, Common fixed point for two pairs of mappings satisfying (E.A) property in generalized metric spaces, Abstr. Appl. Anal.. 2012, Article ID 394830, 15 pages

[18] Z. Mustafa, B. Sims, A new approach to generalized metric spaces, J. Nonlinear Convex Anal.. 7 (2), 289-297 (2006)

[19] Z. Mustafa, H. Obiedat, F. Awawdeh, Some fixed point theorems for mappings on complete G-metric space, Fixed Point Theory Appl. 2008, Article ID 189870, 12 pages

[20] Z. Mustafa, B. Sims, Fixed point theorems for contractive mappings in complete G-metric spaces, Fixed Point Theory Appl.. 2009, Article ID 917175, 10 pages (2009)

[21] Z. Mustafa, W. Shatanawi, M. Bataineh, Existence of fixed points results in G-metric spaces, Int. J. Math. Math. Sci. 2009, Article ID 283028, 10 Pages

[22] Z. Mustafa, M. Khandagu, W. Shatanawi, Fixed point results on complete G-metric spaces, Stud. Sci. Math. Hung.. 48 (3), 304-319 (2011)

[23] Z. Mustafa, Common fixed points of weakly compatible mappings in G-metric spaces, Appl. Math. Sci.. 6 (92), 4589-4600 (2012)

[24] Z. Mustafa, Some new common fixed point theorems under strict contractive conditions in G-metric spaces, J. Appl. Math. 2012, Article ID 248937, 21 pages

[25] Z. Mustafa, H. Aydi, E. Karapinar, On common fixed points in G-metric spaces using (E.A) property, Comput. Math. Appl. 64 (6), 1944-1956 (2012)

[26] V. Popa, A.-M. PATRICIU, A general fixed point theorem for pairs of weakly compatible mappings in G-metric spaces, J. Nonlinear Sci. Appl. 5, 151-160 (2012) .

[27] S. RADENOVIĆ, Remarks on some recent coupled coincidence point results in symmetric Gmetric spaces, Journal of Operators, Volume 20-13, Article ID 290525, 8 pages 
[28] S. Radenović, S. Pantelić, P. SAlimi And J. Vujaković, A note on some tripled coincidence point results in G-metric spaces, International J. of Math. Sci and Engg. Appls, (IJMSEA), Vol. 6 No. VI (November, 2012), pp. 23-38

[29] W. Shatanawi, S. Chauhan, M. Postolache, M.Abbas and S. Radenović, Common fixed points for contractive mappings of integral type in G-metric spaces, J. Adv. Math.Stud. 6 (1) (2013), 53-72.

[30] W. Sintunavarat, P. Kumam, Common fixed point theorems for a pair of weakly compatible mappings in fuzzy metric spaces, J. Appl. Math. 2011, Article ID 637958, 14 pages

[31] H. YE, F. GU, Common fixed point theorems for a class of twice power type contraction maps in G-metric spaces, Abstr. Appl. Anal. 2012, Article ID 736214, 19 pages

[32] Y. YIN, F. GU, Common fixed point theorem about four mappings in G-metric spaces, J. Hangzhou Normal Univ. (Natural Science Edition) 11 (6), 511-515 (2012) 